Document downloaded from:

http://hdl.handle.net/10251/47914

This paper must be cited as:

Law, EL.; Abrahao Gonzales, SM. (2014). Interplay between User Experience (UX) evaluation and system development. International Journal of Human-Computer Studies. 72(6):523-525. doi:10.1016/j.jijhcs.2014.03.003.

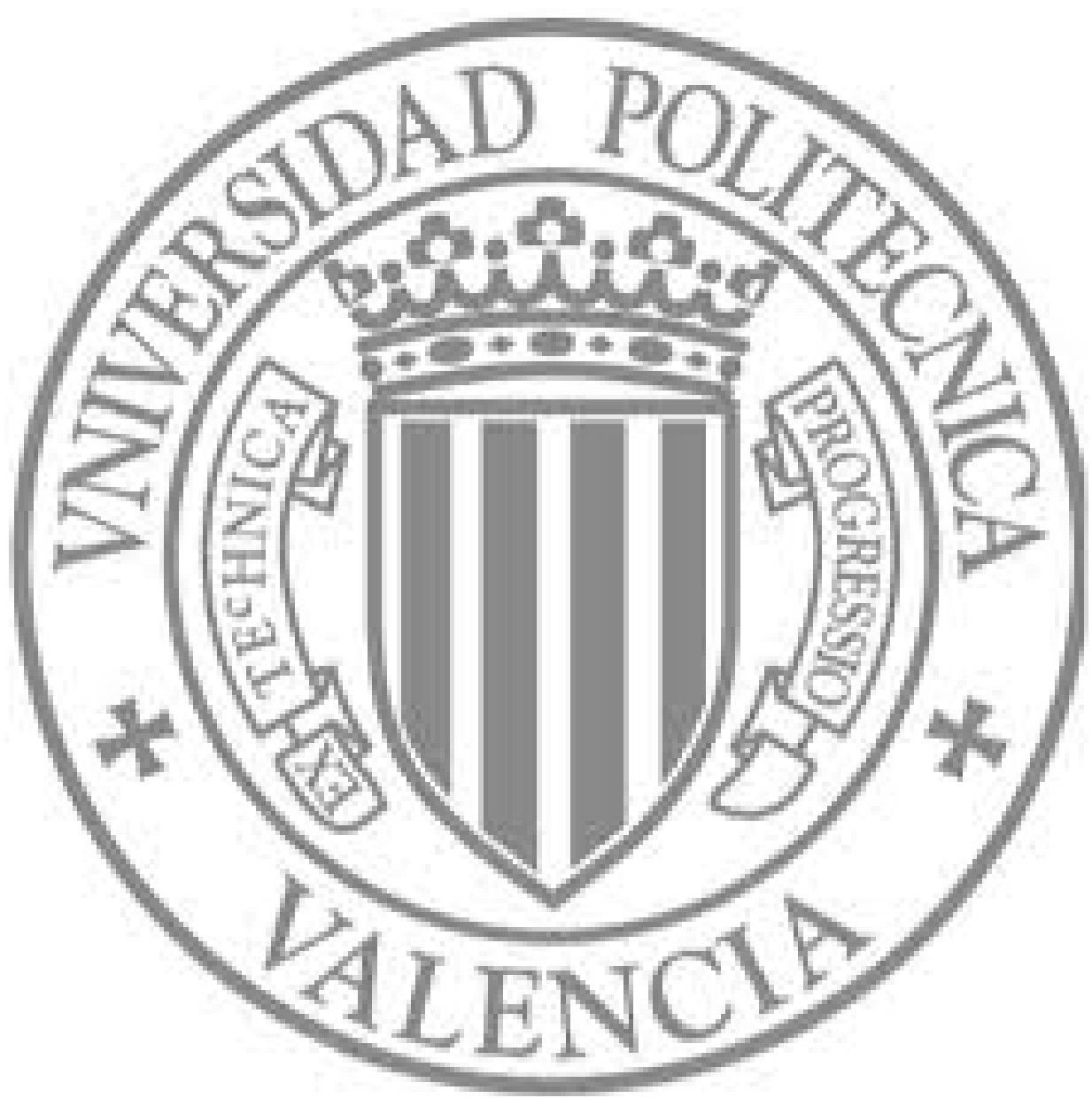

The final publication is available at

http://dx.doi.org/10.1016/j.ijhcs.2014.03.003

Copyright 


\title{
Interplay between User eXperience (UX) Evaluation and System Development
}

\author{
Effie Lai-Chong Law \\ Department of Computer Science \\ University of Leicester \\ United Kingdom \\ elaw@mcs.le.ac.uk
}

\author{
Silvia Abrahão \\ Department of Computer Science \\ Technical University of Valencia \\ Spain \\ sabrahao@dsic.upv.es
}

\begin{abstract}
User Experience (UX) is a maturing research area pertaining to as well as extending beyond the traditional usability. Issues in the realm of usability may be amplified in UX because of its larger scope. Four key nonorthogonal issues are: definition, modeling, method selection, and interplay between evaluation and development. Leveraging the legacy of a series of related research activities, this Special Issue (SI) aims to develop a deeper understanding of how evaluation feedback shapes software development, especially when experiential qualities such as fun, trust, aesthetic values are concerned. Three articles addressing this specific topic from different perspectives and with different approaches are included in this SI.
\end{abstract}

\section{BACKGROUND AND MOTIVATION}

Leveraging the legacy of a series of related research activities (Abrahão et al., 2008; Abrahão et al., 2009; Law, Abrahão \& Stage, 2010) that brought together people from Human-Computer Interaction (HCI) and Software Engineering (SE) communities to discuss the interplay between software evaluation and development, this Special Issue (SI) has further been inspired by more recent insights into the issues pertaining to established usability (e.g. Følstad, Law \& Hornbæk, 2012) as well as maturing User Experience (UX) (e.g., Bargas-Avila \& Hornbæk, 2011; Hassenzahl, 2011).

The shift of emphasis in the field of HCI from usability engineering to a much richer scope of UX where users' emotions, affects, motivations, and values are given as much, if not more, attention than ease of use, ease of learning and basic subjective satisfaction (Law et al., 2009). Among others, four challenges engendered by the new focus of UX are particularly relevant to software development: (i) Definition of UX; (ii) Modelling of UX; (iii) Selection of UX evaluation methods; (iv)Interplay between UX evaluation feedback and software development.

The concept of UX is commonly understood as subjective, context-dependent and dynamic (Law et al., 2009). A formal definition of UX issued by ISO 9241-210: 2010: "A person's perceptions and responses that result from the use and/or anticipated use of a product, system or service" is ambiguous and needs to be refined.

In contrast to usability, UX metrics are yet to be defined. The task is related to ongoing debates on the measurability of experiential qualities (Law, 2011). Both usability and UX measures should enable professionals to benchmark competitive design artefacts and to select right design options. The intriguing question is whether the respective measures have different persuasive power and impact on (re)design and development.

Modelling users' experiences is especially important for understanding, predicting and reasoning about processes of UX with consequences for software design. However, a number of issues pertaining to UX modelling remain to be resolved (Law \& van Schaik, 2010).

Recently, research efforts have been undertaken to collect, consolidate and categorize UX evaluation methods (e.g. Vermeeren et al., 2010). It is envisaged that taxonomies of UX qualities, which can facilitate the selection of UX methods and measures, will come to fruition from these ongoing endeavours.

The first three issues have significant impacts on their fourth one, which is only explored to a limited extent. 


\section{GOALS OF THIS SPECIAL ISSUE}

We understand the relationship between UX and usability as the latter is subsumed by the former. Usability evaluation methods (UEMs) and metrics are relatively more mature (Law, Hvannberg \& Cockton, 2008). In contrast, UX evaluation methods (UXEMs) which draw largely on UEMs (Tullis \& Albert, 2008) are still taking shape. It is conceivable that feeding outcomes of UX evaluation back to the software development cycle to instigate the required changes can even be more challenging than doing so for usability evaluation (UE). It leads to several key issues:

- UX attributes are (much) more fuzzy and malleable, what kinds of diagnostic information and improvement suggestion can be drawn from evaluation data. For instance, a game can be perceived by the same person as a great fun on one day and a terrible boredom the following day, depending on the player's prevailing mood. The waning of novelty effect (cf. learnability differs over time in case of usability) can account for the difference as well. How does the evaluation feedback enable designers/developers to fix this experiential problem (cf. usability problem) and how can they know that their fix works (i.e. downstream utility)?

- Emphasis is put on conducting UE in the early phases of a development lifecycle with the use of low fidelity prototypes, thereby enabling feedback to be incorporated before it becomes too late or costly to make changes (Hertzum, 2006). However, is this principle applicable to UX evaluation? Is it feasible to capture authentic experiential responses with a low-fidelity prototype? If yes, how can we draw insights from these responses?

- The persuasiveness of empirical feedback determines its worth. Earlier research (e.g. Nørgaard \& Hornbæk, 2009) indicates that the development team needs to be convinced about the urgency and necessity of fixing usability problems. Is UX evaluation feedback less persuasive than usability feedback? If yes, will the impact of UX evaluation be weaker than UE?

- The Software Engineering (SE) community has recognized the importance of usability. Efforts are focused on explaining the implications of usability for requirements gathering, software architecture design, and the selection of software components (Juristo, Moreno, \& Sanchez-Segura, 2007). Can such recognition and implications be taken for granted for UX, as UX evaluation methodologies and measures could be very different (e.g. artistic performance)?

- How to translate observational or inspectional data into prioritised usability problems or redesign proposals is thinly documented in the literature. Analysis approaches developed by researchers are applied to a limited extent by practitioners (Følstad, et al., 2012). Such divorce between research and practice could be bitterer in UX analysis approaches, which are essentially lacking.

While the gap between HCI and SE with regard to usability has somewhat been narrowed (e.g. Abrahão et al., 2009), it may be widened again due to the emergence of UX.

The main goal of this SI was to bring together people from HCI and SE to identify challenges and plausible resolutions to optimize the impact of UX evaluation feedback on software development.

The major contribution of this SI to the field of HCI and SE is the understanding of state-of-the-art about the interplay between UX evaluation feedback and system development. Specifically, there are limited studies investigating how different UX evaluation feedback formats such as textual (e.g. diary), audio (e.g. interview), visual (e.g. pictorial scale) and physiological (e.g. eye-tracking) determine their usefulness as well as persuasiveness. Besides, visual and physiological data are more commonly used in UX than in usability, based on the observations that experiences are more difficult to verbalize and more subjective. The role of such evaluation data in system redesign entails further exploration. Besides, there are very few methodological and practical guidelines on integrating UX evaluation and system design in a software development process. The SI will heighten the awareness of the need for more research studies on the above-mentioned issues.

\section{CONTRIBUTIONS}

Three articles representing different perspectives of and approaches to understanding the interplay between UX evaluation and system development are included in this SI. The first article authored by Law and van Schaik aims 
to understand the attitudes of the HCI community towards the measurability of UX constructs - a range of experiential qualities. It is a fundamental concern whether the quantification of a conceptual construct is meaningful and valid. While this issue can be analysed from the epistemological and philosophical perspective, the authors have taken a more pragmatic and empirical approach by conducting questionnaires and interviews with people involved or interested in work related to UX. Results show that the respondents hold diversified views, ranging from very optimistic to very pessimistic about the ease of or even the need of measuring experiential qualities. Above all, the authors emphasize that it is critical to mutually recognize the strengths and weaknesses of different approaches, be they objective physiological measures or subjective accounts of lived experience. Such recognition will not only advance the maturing UX research and but also strengthen the interplay between UX evaluation and system development.

While the first article of this SI investigates the interplay issue more from the conceptual level, the second article examines it more from the practical level. In fact, Ardito and his colleagues query whether the gap between HCI and software engineering with regard to usability has ever been narrowed, although the related efforts have been undertaken more than a decade ago. To address the issue of the persistent gap, Ardito et al conducted an experimental study of which the findings unfortunately confirm their concern that many companies have not paid due attention to the usability and UX practices. Motivated by the goal to bridge the gap, the authors propose an approach called Cooperative Method Development that involves both researchers and practitioners in conducting empirical research. The approach has been proved effective in informing the practitioners why as well as how to improve the software development processes in their companies by taking usability and UX constructs into serious consideration.

The third and last (but the least) article of this SI, authored by Brajnik and Giachin, presents a concrete case how a selection of usability and UX attributes have been evaluated, primarily with established questionnaires. The application context is a touch-screen digital thermometer implemented as mixed-fidelity prototypes of the user interface. The stance adopted by the authors resonates with some of the respondents' views reported in the first article that experiential qualities such as enjoyment, satisfaction, and trust are measurable. The authors, however, qualify the measurability (or the appropriateness to measure) with the fidelity of the interactive product of interest. Specifically, they argue that certain attributes such as subjective mental effort and perceived usability should (though, in principle, still can) not be measured with sketches and storyboards (i.e. low-fidelity prototype), which are deployed to elicit users' evaluation feedback. This supposition is related to the arguments on the predictability of UX aspects (cf. Law, 2011). Besides, the way Brajnik and Giachin classify certain constructs such as perceived ease of use and behavioural intention (i.e., the basic constructs of Technology Acceptance Model [TAM]; Davies et al., 1989) as UX aspects may trigger the recurrent debate on demarcating UX from usability and other software qualities (Roto et al., 2011).

In reflecting the process of coordinating this SI, the number of submissions deemed relevant to the topic of interplay between UX evaluation and system development has been relatively low. It can imply that relevant cases are still under development or yet to be undertaken. In fact, rarely can one find an article that documents a complete cycle of design-evaluation-redesign in detail (cf. the downstream utility of evaluation feedback). We anticipate as well as hope that more studies along this line of inquiry can be available in the near future.

\section{ACKNOWLEDGEMENTS}

Here we would like to express our gratitude to COST Action IC0904 TwinTide (2009-2013) that provided us with precious opportunities to explore the interplay between Human-computer Interaction and Software Engineering. Special thanks should go to Prof. Ebba Thora Hvannberg, University of Iceland and Dr. Arnold P.O.S. Vermeeren, TU Delft, who were the leaders of TwinTide Working Group 3 and 4, respectively. Their generous support and effort in co-organizing the workshop I-UxSED 2012 (NordiCHI 2012, Copenhagen, Denmark), which laid the groundwork for this SI, and in discussing the related topics, are much appreciated. TwinTide enabled not only the two guest editors of this SI but also its partners from 28 European countries to work together over four years. The collaborative experiences have been very inspiring and fruitful. 


\section{REFERENCES}

Abrahão, S., Hornbæk, K., Law, E. L-C., Stage, J. (2009). Interplay between Usability Evaluation and Software Development (I-USED 2009). In Proceedings of INTERACT (2) 2009: 969-970

Abrahão, S., Law, E. L-C., Stage, S., Hornbæk, K., Juristo, N. (2008). Proceedings of the First Workshop on the Interplay between Usability Evaluation and Software Development, Pisa, Italy, September 24, 2008, CEURWS.org 2008

Bargas-Avila, J. A., \& Hornbæk, K. (2011). Old wine in new bottles or novel challenges: a critical analysis of empirical studies of user experience. In Proc. CHI 2011 (pp. 2689-2698).

Davis, F. D. (1989). Perceived usefulness, perceived ease of use, and user acceptance of information technology. MIS Quarterly, 13(3): 319-340.

Følstad, A., Law, L-C., \& Hornbæk, K. (2012): Analysis in practical usability evaluation: a survey study. In Proc. CHI 2012 (pp. 2127-2136).

Hassenzahl, M. (2011): User Experience and Experience Design. In: M. Soegaard, \& R. F. Dam, (eds.), Encyclopedia of Human-Computer $\quad$ Interaction". $\quad$ http://www.interactiondesign.org/encyclopedia/user_experience and experience design.html

Hertzum, M. (2006). Problem Prioritization in Usability Evaluation: From Severity Assessments Toward Impact on Design. International Journal Human Computer Interaction 21(2): 125-146.

Juristo, N., Moreno, A. M., Sanchez-Segura, M. (2007). Guidelines for Eliciting Usability Functionalities. IEEE Transactions on Software Engineering, 33 (11), 744-758.

Law, E. L-C. (2011). The measurability and predictability of user experience. In Proc. EICS 2011 (pp. 1-10).

Law, E. L-C., \& van Schaik. P. (2010). Modelling user experience - an agenda for research and practice. Interacting with Computers, 22(5):313-322.

Law, E. L-C., Abrahão, S., Stage, J. (2010). Proceedings of the First International Workshop on the Interplay between User Experience and Software Development (I-UxSED 2010) (CEUR-WS.org 2010), NordiCHI 2010, Iceland.

Law, E. L-C., Hvannberg, E., \& Cockton, G. (eds.) (2008). Maturing usability: Quality in software, interaction and value. Springer.

Law, E. L-C., Roto, V., Hassenzahl, M., Vermeeren, A., \& Kort, J. (2009). Understanding, scoping and defining user experience: a survey approach. In Proc. CHI 2009 (pp. 719-728).

Nørgaard, M., \& Hornbæk, K (2009). Exploring the Value of Usability Feedback Formats. International Journal Human Computer Interaction 25(1), 49-74.

Roto, V., Law, E. L-C., Vermeeren, A., \& Hoonhout, J. (2010). A White Paper on User Experience. Accessible on: http://www.allaboutux.org/uxwhitepaper

Tullis, T., \& Albert, W. (2008). Measuring the User Experience: Collecting, Analyzing, and Presenting Usability Metrics (Interactive Technologies). Morgan Kaufman.

Vermeeren, A. P.O.S. Law, E. L-C., Roto, V., Obrist, M., Hoonhout, J., Väänänen-Vainio-Mattila, K. (2010). User experience evaluation methods: current state and development needs. In Proc NordiCHI 2010 (pp. 521-530). 